J. Geier ${ }^{1}$

B. M. Hausen ${ }^{2}$

\section{Epikutantestung mit chemisch modifiziertem Kolophonium}

Teil 2: Untersuchungen mit dem Kolophonium-Mix II*

Patch Testing with Modified Colophony Products - Part 2: Studies with the Colophony-Mix II

\section{Zusammenfassung}

In einer 1996 bis 1999 durchgeführten Multicenter-Studie konnte gezeigt werden, dass durch die Testung eines KolophoniumMixes (KM I) mit 3 chemisch modifizierten Kolophonium-Produkten ein erheblicher Anteil zusätzlicher Kolophonium-Allergiker entdeckt wird, der bei alleiniger Testung mit nativem Kolophonium nicht diagnostiziert worden wäre. In den Jahren 2000 und 2001 wurde in 20 dem Informationsverbund Dermatologischer Kliniken (IVDK) angeschlossenen dermatologischen Abteilungen ein zweiter Kolophonium-Mix (KM II) überprüft. Dieser am aktuellen Markt orientierte Mix enthielt drei Bestandteile, nämlich einen Glycerinester, ein Tallharz, und einen Pentaerythritester. Von den 3787 getesteten Patienten reagierten 63 (1,7\%) positiv auf den KM II (15\% Vas.). Mit einem Reaktions-Index von 0,24 und einer Positivity Ratio von 63,5\% hatte die Testzubereitung eine gute diagnostische Trennschärfe. Bei 3740 Patienten wurde der KM II parallel zu Kolophonium (20\% Vas.) aus der Standardreihe getestet. 155 dieser Patienten (4,1\%) reagierten positiv auf das Standard-Kolophonium, und 60 Patienten (1,6\%) auf den KM II. 46 Patienten reagierten auf beide Mixe. 14 Patienten reagierten ausschließlich positiv auf KM II, nicht aber
Abstract

A multicenter study conducted in 1996 to 1999 revealed that patch testing with a mixture of modified colophony products (colophony mix I, CM I) detects a considerable number of patients allergic to colophony, who would have been missed by patch testing only with native colophony from the standard series. In 2000 and 2001, a second colophony mix (CM II) has been patch tested in 20 departments of dermatology joining the Information Network of Departments of Dermatology (IVDK). The composition of this CM II was designed according to the products currently on the market. It contained a glycerol ester, a tall oil resin, and a pentaerythrite ester. Of 3787 patients tested, 63 (1.7\%) reacted positively to CM II (15\% pet.). With a reaction index of 0.24 and a positivity ratio of $63.5 \%$, the CM II had a good diagnostic quality. In 3470 patients, CM II and native colophony from the standard series were tested in parallel. Of these, 155 patients (4.1\%) reacted to colophony, and 60 (1.6\%) to CM II. 46 patients reacted to both test preparations, while 14 had a positive reaction exclusively to CM II, but not to native colophony. These 14 patients represent $8.3 \%$ of the 169 individuals sensitised to colophony in the investigated group. These sensitizations would

* Teil 1: Geier J, Hausen BM: Epikutantestung mit chemisch modifiziertem Kolophonium. Ergebnisse einer Studie der Deutschen Kontaktallergie-Gruppe (DKG) und des Informationsverbundes Dermatologischer Kliniken (IVDK). Dermatologie in Beruf und Umwelt 2000; 48:43-47. An dieser Studie waren die folgenden 20 dermatologischen Abteilungen als Mitglieder der DKG und des IVDK beteiligt (Reihenfolge nach absteigender Patientenzahl): Dortmund (P. J. Frosch, C. Pirker), Essen (U. Hillen), Göttingen (Th. Fuchs, J. Geier), Homburg/Saar (P. Koch), Jena (A. Bauer, W. Wigger-Alberti, M. Kaatz), Dresden

(G. Richter, R. Aschoff), Lübeck (J. Grabbe), Halle (G. Gaber, D. Lübbe), Heidelberg (U. Jappe, M. Hartmann), München Schwabing (M. Agathos), Kiel (J. Brasch), Osnabrück (S. M. John, H.-J. Schwanitz, N. Schürer), Duisburg (J. Schaller), Graz (W. Aberer, B. Kränke), Berlin Charité (T. Zuberbier), Würzburg (J. Arnold, A. Trautmann), Krefeld (M. Lilie, A. Wallerand, S. Wassilew), Ulm (H. Gall, P. Gottlöber, G. Staib), Berlin BWK (A. Köhler), Marburg (I. Effendy, H. Löffler).

Korrespondenzadresse Dr. Johannes Geier · IVDK · Universitäts-Hautklinik Göttingen · von-Siebold-Straße 3 • 37075 Göttingen · E-mail: jgeier@ivdk.org 
auf das Standard-Kolophonium; das sind 8,3\% der insgesamt 169 Kolophonium-Allergiker. Die Sensibilisierungen dieser Patienten wären bei alleiniger Testung mit dem nativen Kolophonium übersehen worden. Es zeigt sich, dass auch weiterhin eine Epikutantestung mit an der aktuellen Marktsituation orientierten Kolophonium-Derivaten sinnvoll ist. have remained undetected if the patients had only been patch tested with native colophony. In conclusion, patch testing with chemically modified colophony products currently on the market continues to be helpful.

\section{Einleitung}

Nach wie vor haben Kolophonium und Kolophonium-Derivate in den verschiedensten Einsatzbereichen eine weite Verbreitung, z. B. in Pflastern, Klebebändern, Dichtungsmassen, Fußbodenklebern, Lacken, Haarentfernungsmitteln, Wachsen, Kaugummi, Karton, Papier u.v.a.m. [1]. Durch die für die industrielle Verwendung vorgenommenen chemischen Modifikationen des Kolophoniums werden nicht nur dessen unerwünschte Eigenschaften reduziert (und gewünschte Eigenschaften verstärkt), sondern auch die sensibilisierenden Eigenschaften verändert [2].

Im Informationsverbund Dermatologischer Kliniken (IVDK) lag die alters- und geschlechts-standardisierte Reaktionsquote auf das in der Standardreihe getestete Kolophonium in den letzten Jahren mit nur geringen Schwankungen um 4,5\% [IVDK, unveröffentlichte Daten]; Kolophonium ist damit nach wie vor eines der allergologisch bedeutendsten Stoffgemische. Die Epikutantestung mit chemisch modifiziertem Kolophonium erwies sich im Rahmen einer 1996 bis 1999 in der Deutschen KontaktallergieGruppe (DKG) und im IVDK durchgeführten Multizenterstudie als eine wichtige zusätzliche diagnostische Maßnahme: Bei der alleinigen Testung mit nativem Kolophonium wären 11,5\% der Kolophonium-Allergiker (67 von 582 sensibilisierten Patienten) unentdeckt geblieben [3]. Damals war dazu ein KolophoniumMix (KM I) überprüft worden, der aus chinesischem Kolophonium, einem Kalkzinkhartharz und einem Maleinsäure-Derivat bestand. In einer Nachfolgeuntersuchung wurde in den Jahren 2000 und 2001 ein weiterer, an den auf dem Markt befindlichen Produkten orientierter Mix mit chemisch modifiziertem Kolophonium, der so genannte Kolophonium-Mix II (KM II), parallel zur Standardreihe epikutan getestet. Insgesamt beteiligten sich 20 dem IVDK angeschlossene dermatologische Abteilungen an der Untersuchung; in 14 dieser Zentren wurde der KM II in der Zeit vom 1. 7.2000 bis 30. 4. 2001 bei 2057 Patienten im Rahmen des IVDK-Allergen-Monitorings (IVDK-Monitorblock) epikutan getestet [4]. Die Daten dieser Patienten sind in die hier vorliegende Auswertung mit eingegangen. Darüber hinaus wurde Daten des Dermatologischen Zentrums Buxtehude (DZB), das dem IVDK nicht angehört, ausgewertet.

\section{Material und Methoden}

Der Kolophonium-Mix II war bereits 1998 im DZB entwickelt und erprobt worden, bevor er auch den dem IVDK angeschlossenen dermatologischen Abteilungen zur Verfügung gestellt wurde. Der im DZB hergestellte Mix (Abb.1-3) enthielt drei Bestandteile, nämlich einen Glycerinester (Sylvatec RE 85, Arizona Chemical AB, Schweden), ein Tallharz (Sylvaros S, Arizona Chemical AB, Schweden), und einen Pentaerythritester (Pentalyn

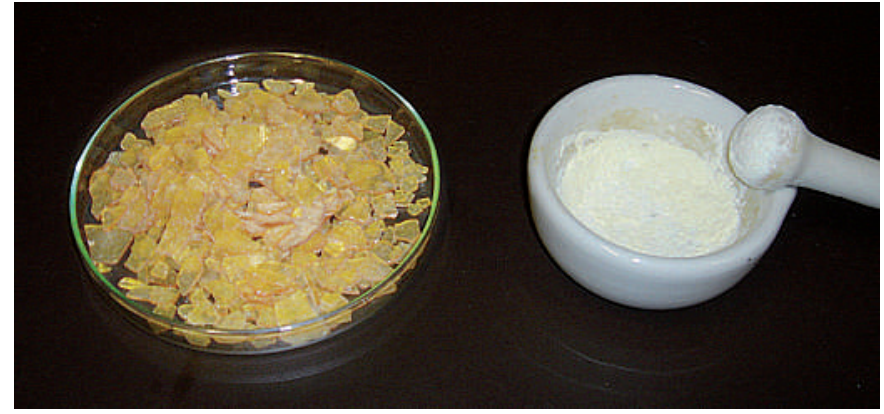

Abb. 1 Kolophoniumderivat im Lieferzustand und nach dem Pulverisieren im Mörser.

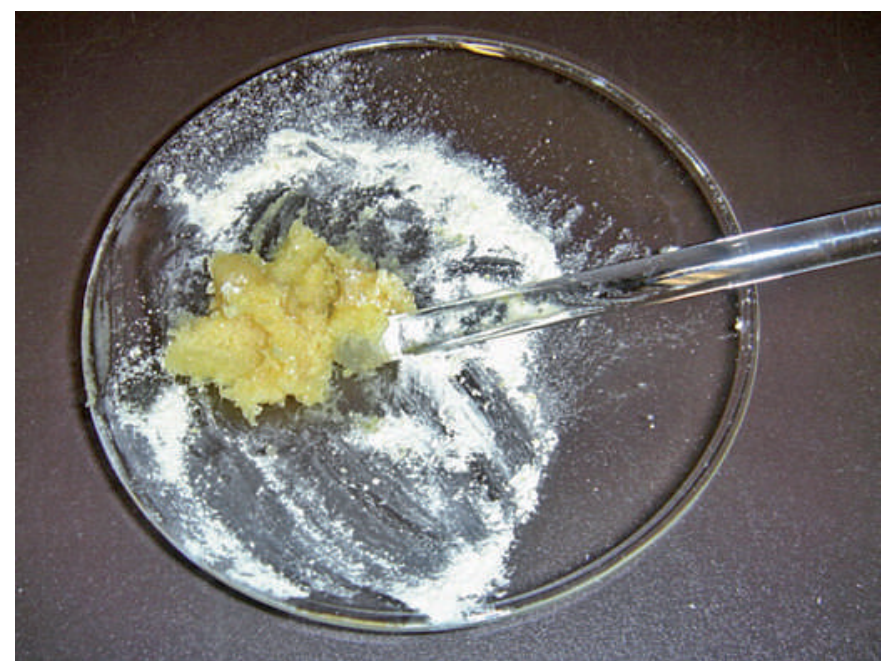

Abb. 2 Anrühren in Vaseline (Verhältnis 1:10).

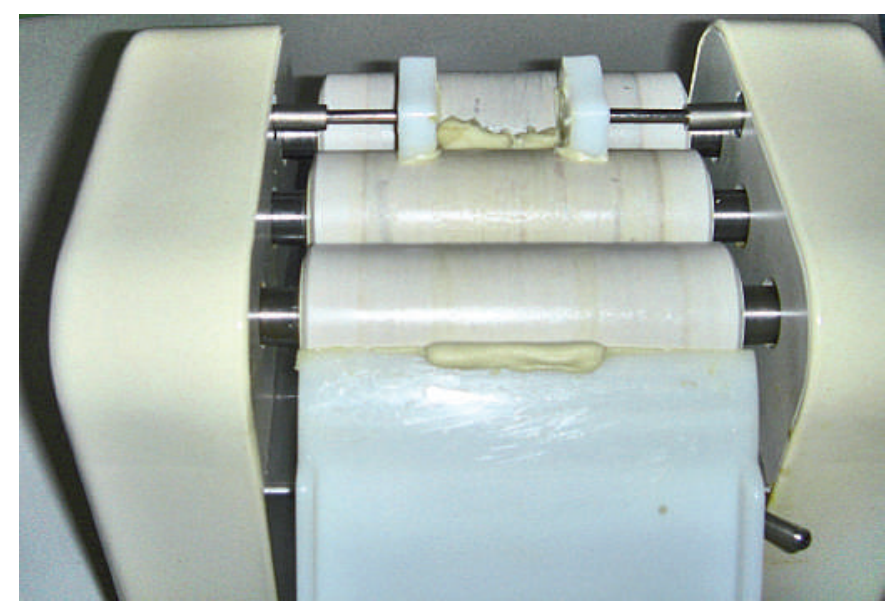

Abb. 3 Homogenisieren des Testmaterials auf dem Dreiwalzenwerk. 
H-E, Hercules, Niederlande). Die Gesamtkonzentration betrug $15 \%$ in Vaseline. Das in der Standardreihe getestete native Kolophonium stammte von Hermal, Reinbek.

In der Zeit vom 1.1.2000 bis zum 31.12.2001 wurde der KM II (15\% Vas.) in den 20 o.g. dermatologischen Abteilungen bei insgesamt 3787 Patienten parallel zur Standardreihe epikutan getestet. Die Epikutantestungen wurden nach den Leitlinien der DKG durchgeführt und abgelesen [5]. Die Expositionsdauer der Tests betrug 1 Tag bei 1548 Patienten (41\%), und 2 Tage bei 2239 Patienten (59\%).

Im DZB selbst wurde der Mix von 1998 bis 2002 bei 2194 Patienten parallel zur Standardreihe und zum Kolophonium-Mix (KM I) [3] epikutan getestet. Die Expositionsdauer des Epikutantests im DZB betrug 1 Tag.

Die Verarbeitung der im IVDK erfassten Testergebnisse und anamnestischen Angaben erfolgte gemäß der IVDK-Routine [6]. Die Daten wurden unter Verwendung des Programmpaketes SAS, Version 8.2., in der Zentrale des IVDK an der UniversitätsHautklinik Göttingen ausgewertet. Es wurden ausschließlich die Testreaktionen der 72-h-Ablesung für die Auswertung berücksichtigt. Die Alters- und Geschlechtsstandardisierung der Reaktionsquoten unterlag den Empfehlungen zu PAFS (patient adjusted frequency of sensitization) [7]. Die Berechnung von Reaktions-Index (RI) und Positivity Ratio (PR) erfolgte gemäß den Original-Formeln [8,9]. Häufigkeitsunterschiede einzelner Merkmale in disjunkten Gruppen wurden mit dem exakten Test von Fisher auf Signifikanz geprüft, wobei als Signifikanzgrenze ein p-Wert von 0,05 angenommen wurde.

\section{Ergebnisse}

Von den 3787 im IVDK getesteten Patienten reagierten 63 (1,7\%) positiv auf den KM II (15\% Vas.). Bei 38 Patienten wurden fragliche Reaktionen beobachtet, bei 1 Patienten eine irritative Reaktion. Der Reaktions-Index lag damit bei 0,24. Bei 40 einfach positiven Reaktionen betrug die Positivity Ratio 63,5\%. Die Testergebnisse sind in Tab. 1 aufgeführt.

Bei 3740 Patienten wurde der KM II parallel zu Kolophonium (20\% Vas.) aus der Standardreihe getestet. Während 60 dieser Patienten (1,6\%) positiv auf den KM II reagierten, zeigten $155 \mathrm{~Pa}-$ tienten (4,1\%) eine positive Reaktion auf das Standard-Kolophonium. 46 Patienten reagierten auf beide Testsubstanzen, das sind 77\% der 60 Patienten mit Reaktion auf KM II bzw. 30\% der 155 Patienten mit Reaktion auf das Standard-Kolophonium. 14 Patienten reagierten ausschließlich positiv auf KM II, nicht aber auf das Standard-Kolophonium; das sind 8,3\% der insgesamt 169 Kolophonium-Allergiker unter diesen 3740 Patienten. Eine Übersicht mit den dichotomisierten Testreaktionen auf KM II und Standard-Kolophonium gibt Tab. 2 wieder.

Der in der früheren Studie untersuchte Kolophonium-Mix (KM I) und der KM II wurden bei nur 181 Patienten parallel getestet, von denen 5 auf den KM II und 1 auf KM I positiv reagierten. Gemeinsame Reaktionen auf beide Mixe wurden nicht beobachtet.
Tab. 1 Testreaktionen an Tag 3 auf Kolophonium-Mix II (15\% Vas.)

\begin{tabular}{|lrr|}
\hline Reaktion & Anzahl Patienten & Prozent \\
\hline negativ & 3685 & 97,3 \\
\hline$?$ & 38 & 1,0 \\
+ & 40 & 1,1 \\
++ & 20 & 0,5 \\
\hline+++ & 3 & 0,1 \\
\hline irritativ & 1 & 0,0 \\
\hline Summe & 3787 & 100,0 \\
\hline
\end{tabular}

Tab. 2 Testreaktionen an Tag 3 auf Kolophonium-Mix II (15\% Vas.) und Kolophonium (20\% Vas.) aus der Standardreihe bei 3740 Patienten

\begin{tabular}{|llccc}
\hline & \multicolumn{3}{c}{ Kolophonium } \\
\hline & & neg., ?, irr. & positiv & Summe \\
\hline \multirow{2}{*}{ Kolophonium-Mix II } & neg., ?, irr. & 3571 & 109 & 3680 \\
& positiv & 14 & 46 & 60 \\
& Summe & 3585 & 155 & 3740 \\
\hline
\end{tabular}

Tab. 3 MOAHLFA-Index der Patienten mit positiver $(n=63)$ und negativer $(n=3685)$ Reaktion auf den Kolophonium-Mix II

\begin{tabular}{llll}
\hline & & Positive (n=63) & Negative $(\mathbf{n = 3 6 8 5})$ \\
\hline Männlich & M & $36,5 \%$ & $40,3 \%$ \\
\hline Berufsdermatose & O & $20,6 \%$ & $15,7 \%$ \\
Atopische Dermatitis & A & $11,1 \%$ & $16,3 \%$ \\
\hline Handekzem & H & $30,2 \%$ & $28,6 \%$ \\
\hline Beinekzem & L & $20,6 \%$ & $10,3 \%$ \\
\hline Gesichtsekzem & F & $14,3 \%$ & $14,0 \%$ \\
\hline Alter $\geq 40$ Jahre & A & $71,4 \%$ & $65,7 \%$ \\
\hline
\end{tabular}

Die klinische Relevanz der positiven Reaktionen auf den KM II war im individuellen Einzelfall in der klinischen Routine kaum zu klären. Um aus den epidemiologischen Daten Hinweise für eine mögliche Exposition zu bekommen, wurden die erfassten anamnestischen Daten der Patienten mit positiver und negativer Reaktion auf den KM II verglichen. In Tab. 3 sind die im MOAHLFA-Index zusammengefassten Punkte dargestellt. Signifikante Unterschiede bestanden nur beim Anteil der Patienten mit BeinEkzem (20,6\% bei den KM-II-Positiven vs. 10,3\% bei den KM-IINegativen; $p=0,02$ ). Die prozentuale Verteilung aller anderen erfassten anamnestischen Angaben - auch über den MOAHLFAIndex hinaus - unterschied sich nicht signifikant.

Von den 2194 im DZB 1998 bis 2002 getesteten Patienten reagierten 76 (3,5\%) auf das Kolophonium in der Standardreihe, 15 $(0,7 \%)$ auf den KM II und 44 (2,0\%) auf den KM I. 7 Patienten reagierten ausschließlich auf den KM II und 2 Patienten auf beide Kolophonium-Mixe. 
Wie schon bei der ersten Untersuchung mit chemisch modifiziertem Kolophonium lag auch bei der jetzigen Testung mit dem KM II die Reaktionsquote auf das modifizierte Kolophonium deutlich niedriger als die auf das native Kolophonium. Dies ist nicht verwunderlich, da es sich bei den Kolophonium-Mixen um spezielle Produkte handelte, deren allergene Eigenschaften durch die chemische Modifikation verändert wurden. Die Mixe KM I und KM II sollten ja auch nicht das native Kolophonium in der Diagnostik ersetzen, sondern die diagnostische Palette bei entsprechenden Patienten erweitern. Dass dies gelungen ist, zeigt der Zugewinn von 67 zusätzlich diagnostizierten Patienten in der ersten Untersuchung (11,5\% der damals insgesamt 582 Kolophonium-Allergiker), die ohne die Testung mit dem KM I übersehen worden wären. Ähnliches lässt sich auch beim KM II feststellen: Hier waren es 14 Patienten (8,3\% der insgesamt 169 Kolophonium-Allergiker), die ausschließlich auf den KM II positiv reagierten, deren Kolophonium-Allergie also bei alleiniger Testung mit dem nativen Kolophonium in der Standardreihe nicht diagnostiziert worden wäre.

Der relativ hohe Reaktions-Index und die niedrige Positivity Ratio des KM II zeigen, dass hier eine Testzubereitung mit günstigen diagnostischen Eigenschaften vorlag. Gleichzeitig wird deutlich, dass die beobachteten positiven Reaktionen tatsächlich allergische Reaktionen waren, und nicht vermehrt falsch positive Befunde aufgetreten sind. Hierfür spricht auch, dass unter den Patienten mit positiver Reaktion auf KM II signifikant mehr BeinPatienten waren, denn der im KM II enthaltene Pentaerythritester (Pentalyn H-E) wird häufig in Hydrokolloidverbänden für die Versorgung von Ulcera crurum verwendet. Er ist als ätiologisches Agens beschrieben worden [ 10 - 13]. Insofern ist - auch ohne den expliziten Allergennachweis in jedem Einzelfall - das Ergebnis unserer Untersuchung plausibel.

Sowohl die erste Untersuchung aus dem Jahre 2000 [3] als auch die jetzige Studie machen deutlich, dass es notwendig ist, die Testung des nativen Kolophoniums in der Standardreihe durch ein Gemisch mit modifizierten Kolophoniumprodukten zu ergänzen. In den beiden getesteten Kolophonium-Mixen waren insgesamt 6 verschiedene Produkte enthalten. Da auf dem Markt jedoch weit mehr als 100 verschiedene Kolophoniumprodukte eingesetzt werden, wird es wohl nie zu verwirklichen sein, ein Gemisch zusammenzustellen, das alle Kolophoniumsorten erfasst. Einerseits lassen sich nur wenige Sorten wegen der notwendig hohen Konzentration in einem Gemisch zusammenzufassen, andererseits ändert sich das Angebot an verwendeten Sorten ständig, so dass man gezwungen wäre, sich durch Wechsel des Testmaterials den Veränderungen anzupassen. Schon bei der Erstellung des KM II wurde offensichtlich, dass mehr als drei Derivate nicht in einem Gemisch zu vereinigen sind. Dieser Mix enthielt z.B. einen der neueren „Klebrigmacher“ („tackifier“). Dieser erwies sich als so klebrig, dass nicht nur das Mischen zu einem Problem wurde, sondern auch das Einbringen in die Testspritzen. Die anschließende Reinigung des Dreiwalzenwerkes (Abb.3), der verwendeten Werkzeuge (z.B. der Spatel) und des
Labors zog sich über eine Woche hin. Dies war auch der Grund, warum die Verwendung des KM II für die weitere routinemäßige Epikutantestung wieder aufgegeben wurde. Einfacher wäre es, eine Testreihe mit möglichst vielen marktgängigen modifizierten Produkten aufzubauen, die zum Einsatz käme, wenn der begründete Verdacht einer Kolophoniumallergie vorliegt, das Standardkolophonium aber keine positive Reaktion gezeigt hat.

Eine derartige, vom Autor B. M. H. seit Jahren eingesetzte Testreihe mit 37 modifizierten Kolophoniumprodukten zeigte in der Mehrzahl der Fälle stark positive Reaktionen auf 35 der getesteten Derivate, während 2 der hydrierten Produkte nur schwache Reaktionen ergaben oder negativ blieben. Dieses Ergebnis steht in guter Übereinstimmung mit dem experimentell bestimmten, sehr niedrigen Sensibilisierungsvermögen dieser Produkte [2], wie z. B. einem kommerziellen und einem selbst gegossenen Geigenharz [14].

\section{Literatur}

${ }^{1}$ Hausen BM. Kolophonium. In: Hausen BM, Brinkmann, Dohn (Hrsg). Lexikon der Kontaktallergene, 6. Ergänzungslieferung, K 4. Landsberg: Ecomed, 1998: 1 - 15

${ }^{2}$ Hausen BM, Mohnert J. Contact allergy due to colophony. Part V: Patch test results with different types of colophony and modified-colophony products. Contact Dermatitis 1989; 20: 295-301

${ }^{3}$ Geier J, Hausen BM. Epikutantestung mit chemisch modifiziertem Kolophonium. Ergebnisse einer Studie der Deutschen KontaktallergieGruppe (DKG) und des Informationsverbundes Dermatologischer Kliniken (IVDK). Dermatologie in Beruf und Umwelt 2000; 48: 43-47

${ }^{4}$ Aberer W, Komericki P, Uter W, Hausen BM, Lessmann H, Kränke B, Geier J, Schnuch A. Epidemiologische Überwachung von Kontaktallergenen. Der „Monitorblock“ des IVDK. Hautarzt 2003; 54: 741 - 749

${ }^{5}$ Schnuch A, Aberer W, Agathos M, Brasch J, Frosch PJ, Fuchs Th, Richter $G$ für die Deutsche Kontaktallergie-Gruppe. Leitlinien der Dt. Dermatologischen Gesellschaft (DDG) zur Durchführung des Epikutantests mit Kontaktallergenen. Hautarzt 2001; 52: 864-866

${ }^{6}$ Uter W, Schnuch A, Geier J, Frosch PJ. Epidemiology of contact dermatitis. The Information Network of Departments of Dermatology (IVDK) in Germany. European Journal of Dermatol 1998; 8: 36-40

7 Schnuch A. PAFS : population-adjusted frequency of sensitization (I). Influence of sex and age. Contact Dermatitis 1996; 34: 377-382

${ }^{8}$ Brasch J, Henseler T. The reaction index: a parameter to assess the quality of patch test preparations. Contact Dermatitis 1992; 27: $203-$ 204

${ }^{9}$ Geier J, Uter W, Lessmann H, Schnuch A. The positivity ratio - another parameter to assess the diagnostic quality of a patch test preparation. Contact Dermatitis 2003; 48: 280-282

${ }^{10}$ Downs AMR, Sharp LA, Sansom JE. Pentaerythritol-esterified gum rosin as a sensitizer in Granuflex ${ }^{\circledR}$ hydrocolloid dressing. Contact Dermatitis 1999; 41: 162 - 163

${ }^{11}$ Hausen BM, Kulenkamp D. Allergische Kontaktdermatitis auf einen Hydrokolloidverband bei Kolophoniumallergikern. Aktuelle Dermatologie 1998; 24: $174-177$

${ }^{12}$ Sasseville D, Tennstedt D, Lachapelle JM. Allergic contact dermatitis from hydrocolloid dressings. American Journal of Contact Dermatitis 1997; 8: 236-238

${ }^{13}$ Schliz M, Rauterberg A, Weiß J. Allergic contact dermatitis from hydrocolloid dressings. Contact Dermatitis 1996; 34: 146

${ }^{14}$ Hausen BM. Ein Geigenharz mit geringer Sensibilisierungspotenz. Praktische Auswirkungen der experimentellen Untersuchungen zur Sensibilisierungspotenz von Kolophonium-Derivaten. Aktuelle Dermatologie 1990; 16: $32-33$ 